\title{
Acute effects of instillation of surfactant in severe respiratory distress syndrome
}

\author{
H L HALLIDAY, F B McCORD, B G McCLURE, AND M McC REID \\ Department of Child Health, The Queen's University of Belfast and Royal Maternity Hospital, Belfast
}

SUMmARY Doppler ultrasound measurements of pulmonary blood flow in 20 babies with severe respiratory distress syndrome treated in a randomised controlled trial of surfactant replacement showed that the immediate improvement of oxygenation was not associated with a significant increase in pulmonary blood flow. Reduction in ventilator settings and increases in the extent of chest wall movements measured by a cardiorespiratory monitor suggested that the improvement after surfactant had been given was a result of alveolar stabilisation and increased pulmonary compliance. Further simultaneous studies of pulmonary blood flow and pulmonary compliance are needed to confirm these findings.

Natural surfactant has been used to reverse the effects of respiratory failure in animals. ${ }^{1-5}$ These studies showed an almost instantaneous increase in arterial oxygen tension followed by a more gradual change in $\mathrm{pH}$ and arterial carbon dioxide tension. This rapid improvement in oxygenation was reminiscent of the action of tolazoline in babies with pulmonary hypertension, and we wondered if surfactant acted like a pulmonary vasodilator drug.

In lambs changes in pulmonary compliance can be detected 15-30 minutes after giving surfactant $t^{1-3}$ but pulmonary blood flow either does not change $e^{4}$ or increases after a delay of about two to three hours. ${ }^{5}$ In immature baboons both compliance and pulmonary blood flow increase after instillation of surfactant, pulmonary blood flow by threefold. ${ }^{6}$ As far as we are aware there have been no studies of pulmonary blood flow changes in babies after surfactant treatment.

\section{Patients and methods}

Based on the threefold increase in pulmonary blood flow in immature baboons after surfactant treatment ${ }^{6}$ we estimated that to detect a similar change in babies with a power of $80 \%$ and a probability of $<0.05$ (two tailed test) would require a sample of 20 patients.

Serial measurements of pulmonary blood flow were made in 20 consecutive babies who were taking part in a randomised controlled trial of surfactant replacement. $^{7}$ All babies entering the study had severe respiratory distress syndrome requiring mechanical ventilation with $>60 \%$ oxygen when less than 15 hours old. ${ }^{7}$

The surfactant was isolated from porcine lungs by chloroform-methanol extraction and further purified by liquid-gel chromatography ${ }^{8}$; it was given in a total dose of $200 \mathrm{mg} / \mathrm{kg}(2.5 \mathrm{ml} / \mathrm{kg})$ through the endotracheal tube. Treated babies were disconnected from the ventilator and $1.25 \mathrm{ml} / \mathrm{kg}$ of surfactant was instilled into each main bronchus through a sterile feeding tube. After each instillation the baby was manually ventilated at a rate $40-60$ / minute for one minute using the same oxygen concentration and pressure of $<35 \mathrm{~cm} \mathrm{H}_{2} \mathrm{O}$ before being reconnected to the ventilator without altering its settings. In the babies who acted as controls, manual ventilation without instillation of liquid was carried out. After enrolment into the study blood gases were measured at five, 15,30 , and 60 minutes, and appropriate changes in the ventilator settings were made to keep arterial oxygen tension about $7 \mathrm{kPa}$, arterial carbon dioxide tension about $6 \mathrm{kPa}$ and $\mathrm{pH}$ greater than 7.3. The protocol required that the inspired oxygen concentration should be lowered first, and then the inspiratory pressure, the frequency, and the inspiratory: expiratory time ratio. ${ }^{7}$

Pulmonary blood flow was measured 15 minutes, one and four hours after instillation of surfactant with an ATL Mk V Duplex scanner. Pulmonary blood flow is difficult to measure directly but is equivalent to pulmonary venous return, which can be measured as flow across the aortic valve if there are no intracardiac shunts. ${ }^{9}$ Such intracardiac shunts were excluded 
in all study babies by careful cross sectional and pulsed Doppler echocardiographic examinations.

Alveolar oxygen tension was calculated as previously described using the equation: ${ }^{7}$ alveolar oxygen tension $=$ fraction of inspired oxygen $\times 94-$ arterial carbon dioxide tension $/ 0 \cdot 8 \mathrm{kPa}$. The ratio of arterial:alveolar oxygen tension was taken as a measure of oxygenation as it is not influenced by the amount of inspired oxygen. We used the product of mean airway pressure and ventilator rate as a measure of ventilation. If arterial carbon dioxide tension remains constant and spontaneous respirations are largely absent, the product of mean arterial pressure $\times$ ventilator rate should be a measure of ventilation that correlates inversely with compliance. ${ }^{10}$

Respiration traces were obtained from a Corometrics 512 monitor before, during, and after surfactant replacement. This monitor measures the extent of chest wall movement by recording changes in transthoracic impedance, which correlate indirectly with pulmonary compliance. ${ }^{11}$

The significance of differences between the groups was assessed by the Student's $t$ test. In addition, $95 \%$ confidence intervals were calculated for the differences in mean values between treated and control babies. ${ }^{12}$

\section{Results}

The 12 treated babies had a mean (SD) birth weight of 1335 (312) g and gestational age $28 \cdot 4(1 \cdot 1)$ weeks. The median age at enrolment was 5 hours (range 2-13). The eight control infants had a mean birth weight of 1401 (258) $\mathrm{g}$ and gestational age $28 \cdot 8(1 \cdot 9)$ weeks; their median age at enrolment was 7 hours (range 2-15). Fifteen babies were given muscle relaxants during ventilation (eight treated and seven controls).

Fig 1 compares the changes in arterial to alveolar oxygen tension ratio with the calculated measure of mechanical ventilation and pulmonary blood flow in $\mathrm{ml} / \mathrm{kg} /$ minute. There was an immediate increase in arterial:alveolar oxygen tension ratio so that treated babies had significantly higher ratios from $15 \mathrm{~min}$ utes to four hours (fig 1 and table). The table shows positive values for $95 \%$ confidence intervals of the difference between treated and control babies at 15 minutes and one hour. The product of mean arterial pressure $\times$ ventilator rate was significantly lower for treated babies by 15 minutes and remained so for the four hour period (fig 1) but wide confidence intervals permit only the four hour difference to be confidently accepted as applying to a larger population of babies (table). Pulmonary blood flow was not significantly different between treated and control infants and did not change after treatment with surfactant. 95\% Confidence intervals of the difference between treated and control babies are wide for this measurement (table). Arterial carbon dioxide tensions and $\mathrm{pH}$ were similar in each group and did not change significantly after instillation of surfactant.

Fig 2 shows an example of a respiratory trace from the Corometrics 512 monitor of a baby before.
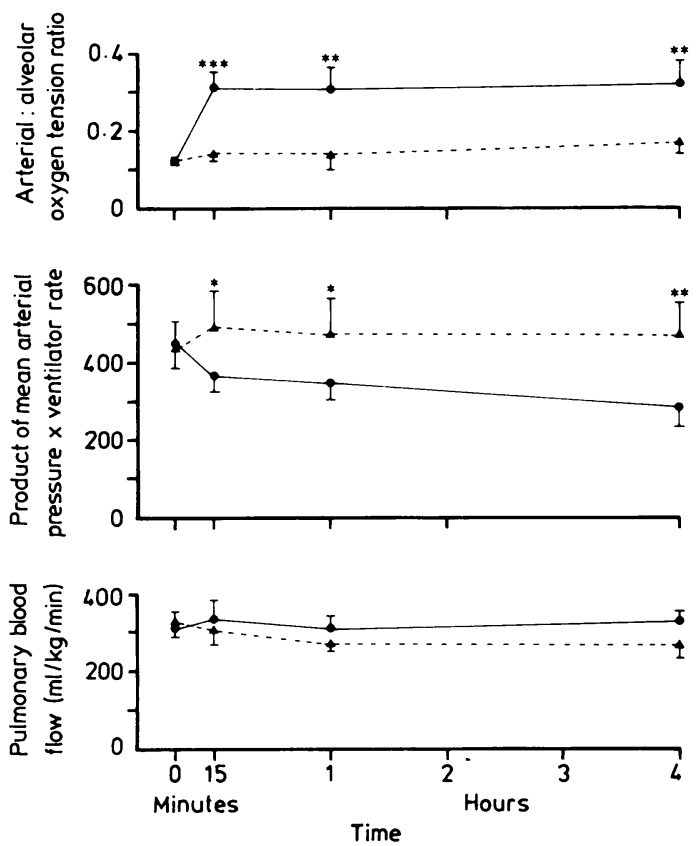

Fig 1 Comparison of arterial:alveolar oxygen tension ratio, product of mean airway pressure $\times$ ventilator rate, and pulmonary blood flow for treated (-) and control ( $\mathbf{\Delta}----\mathbf{\Delta})$ babies after treatment with surfactant. Time 0 indicates values just before instillation of surfactant. Mean (SE), ${ }^{*} p<0 \cdot 05,{ }^{* *} p<0 \cdot 01,{ }^{* * *} p<0 \cdot 001$.

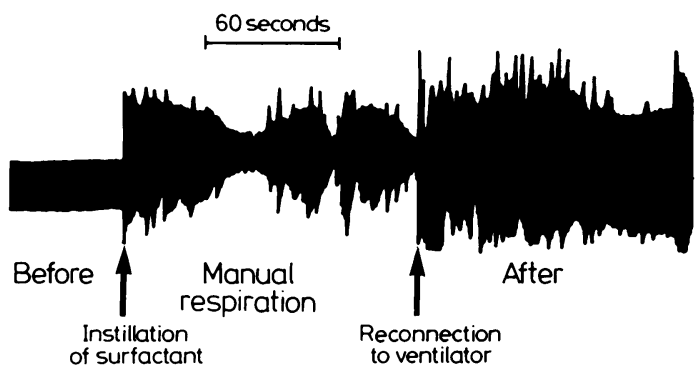

Fig 2 Respiratory trace from Corometrics 512 monitor before, during, and after instillation of surfactant in a baby with severe respiratory distress syndrome. 
during, and after replacement of surfactant. The extent of chest wall movement (transthoracic impedance) increased immediately after surfactant had been given, and was maintained after the baby was reconnected to the ventilator. This pattern was seen in nearly all the treated babies, but similar changes were not seen in control babies.

\section{Discussion}

Natural surfactants have been shown to improve oxygenation in animals ${ }^{1-5}$ and in babies. ${ }^{78}$ The rise in arterial oxygen tension occurs immediately and precedes the decrease in arterial carbon dioxide tension and the increase in compliance. ${ }^{3}$ Jacobs et al found that the surface tension of alveolar washings correlated better with arterial oxygen tension than with arterial carbon dioxide tension or tidal volume. $^{3}$ Vidyasagar et al showed a threefold increase in pulmonary blood flow within three hours of surfactant replacement in 15 immature baboons with severe respiratory distress syndrome. ${ }^{6}$ Pulmonary compliance also increased and the authors attributed the improvement in oxygenation to this, and the change in pulmonary blood flow to the presence of a left to right shunt across a patent ductus arteriosus. ${ }^{6}$

We speculated that increase in pulmonary blood flow might be responsible for the improved oxygenation as it amounted to about a $300 \%$ increase over baseline values. We estimated that a study of 20 infants would be large enough to detect a similar threefold increase in pulmonary blood flow.
That we were unable to show such an increase may be explained in one of four ways. Firstly, baboons with respiratory distress syndrome do not behave like babies with respiratory distress syndrome, and secondly that the methods of measuring pulmonary blood flow were different; Vidyasagar et al used a microsphere technique ${ }^{6}$ and we used a pulsed Doppler ultrasound method. Thirdly, the surfactant preparations are not identical and clinical studies with our porcine surfactant did not show an increased incidence of patent ductus arteriosus. ${ }^{7}$ An alternative explanation is that we entered too few babies into our study to show a change in pulmonary blood flow. If the change had been threefold, however (that is, of the same order as the change in oxygenation), then our study should have been able to detect this. Our results are consistent with a modest change in pulmonary blood flow, certainly much less than the change in oxygenation.

We believe that the acute change in oxygenation is caused by improvement in the ventilation:perfusion ratio and not to an immediate increase in pulmonary blood flow. Our findings suggest that improved compliance is a more likely explanation and confirm those of Jobe et al who measured cardiac output and pulmonary blood flow before and after treatment of premature lambs with surfactant and found no difference. ${ }^{4}$ We did not make direct measurements of pulmonary compliance, and have assumed that it changed with reduced ventilator settings and alterations in the cardiorespiratory trace of transthoracic impedance. Daily et al showed an inverse correlation between compliance and

Table Difference between control and treated babies in arterial:alveolar oxygen tension ratio, mean airway pressure $\times$ ventilation rate, and pulmonary blood flow before and after instillation of surfactant

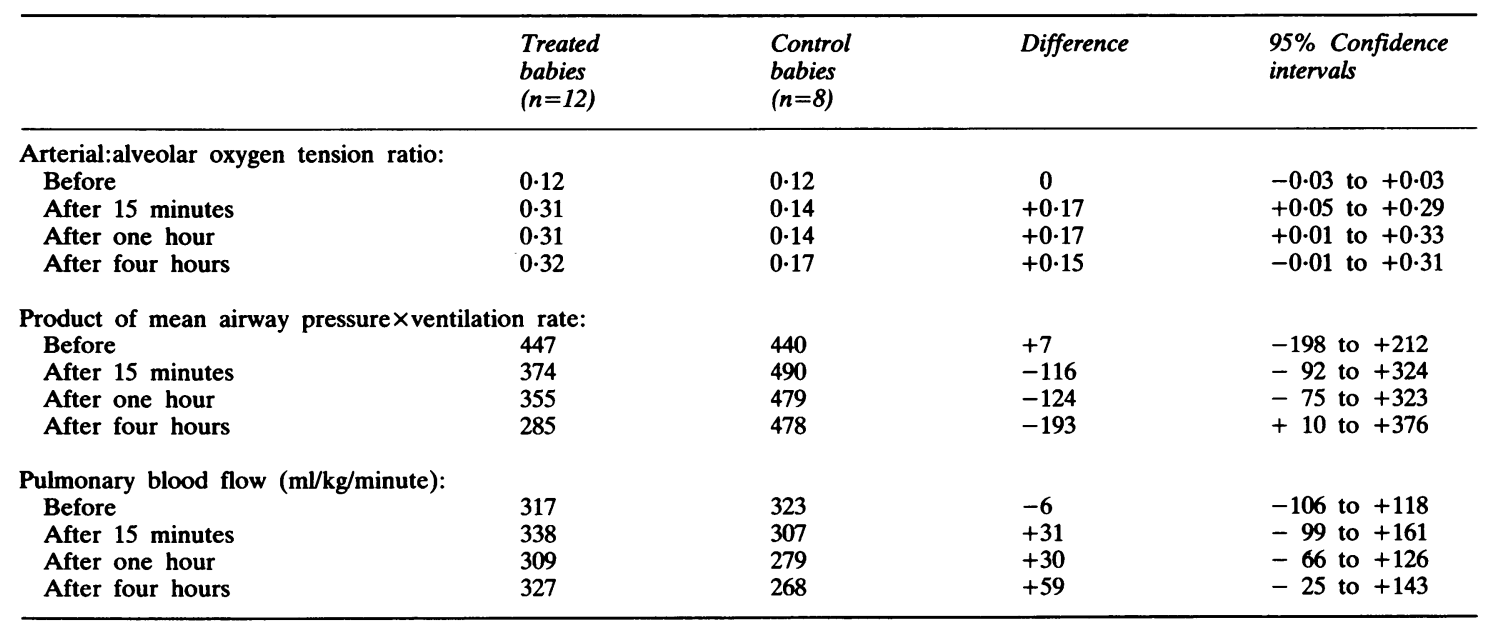


transthoracic impedance. ${ }^{11}$ Further studies are needed of direct measurement of lung compliance in babies having surfactant replacement. Simultaneous measurements of lung compliance and pulmonary blood flow would help to clarify the mechanisms of the acute effect of exogenous surfactant and may also help to predict the long term response of individual babies.

The surfactant was prepared in Stockholm by Dr T Curstedt and Dr B Robertson, supported by the Swedish Medical Research Council (Project number 3351) and the Swedish National Association against Heart and Chest Diseases. Dr FB McCord was supported by a grant from the Perinatal Trust Fund of Northern Ireland. We thank Lynda Thompson for typing the manuscript.

\section{References}

${ }^{1}$ Jobe A, Ikegami M, Glatz T, Yoshida Y, Diakomanolis E, Padbury J. Duration and characteristics of treatment of premature lambs with natural surfactant. J Clin Invest 1981;67:370-5.

2 Jobe A, Ikegami M, Jacobs $\mathbf{H}$, Jones S. Surfactant and pulmonary blood flow distributions following treatment of premature lambs with natural surfactant. $J$ Clin Invest 1984;73:848-56

3 Jacobs H, Jobe A, Ikegami M, Glatz T, Jones SJ, Barajas L. Premature lambs rescued from respiratory failure with natural surfactant: clinical and biophysical correlates. Pediatr Res 1982;16:424-9.

${ }^{4}$ Jobe A, Jacobs H, Ikegami M, Jones S. Cardiovascular effects of surfactant suspensions given by tracheal instillation to premature lambs. Pediatr Res 1983;17:444-8.

5 Clyman RI, Jobe A, Heymann M, et al. Increased shunt through the patent ductus arteriosus after surfactant replacement therapy. J Pediatr 1982;100:101-7.

- Vidyasagar D, Maeta H, Raju TN, et al. Bovine surfactant (surfactant TA) therapy in immature baboons with hyaline membrane disease. Pediatrics 1985;75:1132-42.

${ }^{7}$ McCord FB, Curstedt T, Halliday HL, McClure G, Reid $\mathrm{MMcC}$, Robertson B. Surfactant treatment and incidence of intraventricular haemorrhage in severe respiratory distress syndrome. Arch Dis Child 1988;63:10-6.

${ }^{8}$ Noack G, Berggren $P$, Curstedt $T$, et al. Severe neonata respiratory distress syndrome treated with the isolated phopholipid fraction of natural surfactant. Acta Paediatr Scand 1987;76:697-705.

9 Alverson DC, Eldridge M, Dillon T, Yabek SM, Berman W Jr. Non-invasive pulsed Doppler determination of cardiac output in neonates and children. $J$ Pediatr 1982;101:46-50.

10 Bohn DJ, James I, Filler RM, et al. The relationship between $\mathrm{PaCO}_{2}$ and ventilation parameters in predicting survival in congenital diaphragmatic hernia. J Pediatr Surg 1984;19:666-71.

1 Daily W, Olsson T, Victorin L. Transthoracic impedance: V. Effects of early and late clamping of the umbilical cord with special reference to the ratio air-to-blood during respiration. Acta Paediatr Scand 1970;207(suppl):57-72.

12 Gardner MJ, Altman DG. Confidence intervals rather than $p$ values: estimation rather than the hypothesis testing. $\mathrm{Br} \mathrm{Med} \mathrm{J}$ 1986;292:746-50.

Correspondence to Dr HL Halliday, Neonatal Intensive Care Unit, Royal Maternity Hospital, Belfast BT12 6BB, Northern Ireland.

Accepted 28 July 1988 